\title{
Sarcopenia and visceral adiposity predict poor overall survival in hepatocellular carcinoma patients after curative hepatic resection
}

\author{
Hee Yoon Jang ${ }^{1 \#}$, Gwang Hyeon Choi ${ }^{1 \#}$, Sung Ho Hwang ${ }^{1 \wedge}$, Eun Sun Jang ${ }^{1 \wedge}$, Jin-Wook Kim ${ }^{1 \wedge}$, \\ Joong Mo Ahn ${ }^{2}$, Youngrok Choi ${ }^{3}$, Jai Young Cho ${ }^{3}$, Ho-Seong Han ${ }^{3}$, Jaebong Lee ${ }^{4}$, Jung Wha Chung ${ }^{5} \wedge$, \\ Joo Yeong Baeg ${ }^{6}$, Sook-Hyang Jeong ${ }^{1 \wedge}$
}

${ }^{1}$ Department of Internal Medicine, Seoul National University Bundang Hospital, Seoul National University College of Medicine, Seongnam-si, Gyeonggi-do, Republic of Korea; ${ }^{2}$ Department of Radiology, Seoul National University Bundang Hospital, Seoul National University College of Medicine, Seongnam-si, Gyeonggi-do, Republic of Korea; ${ }^{3}$ Department of Surgery, Seoul National University Bundang Hospital, Seoul National University College of Medicine, Seongnam-si, Gyeonggi-do, Republic of Korea; ${ }^{4}$ Division of Statistics, Medical Research Collaborating Center, Seoul National University Bundang Hospital, Seoul National University College of Medicine, Seongnam-si, Gyeonggi-do, Republic of Korea; ${ }^{5}$ Department of Internal Medicine, Wonkwang University Sanbon Hospital, Gunpo-si, Gyeonggi-do, Republic of Korea; ${ }^{6}$ Department of Internal Medicine, Sheikh khalifa Speciality Hospital, Ras al Khaimah, United Arab Emirates

Contributions: (I) Conception and design: SH Jeong, JM Ahn; (II) Administrative support: None; (III) Provision of study materials or patients: SH Jeong, ES Jang, JW Kim, Y Choi, JY Cho, HS Han; (IV) Collection and assembly of data: HY Jang, GH Choi, SH Hwang, ES Jang, JW Kim, Y Choi, JY Cho, HS Han, JW Chung, JY Baeg, SH Jeong; (V) Data analysis and interpretation: HY Jang, GH Choi, SH Jeong, J Lee; (VI) Manuscript writing: All authors; (VII) Final approval of manuscript: All authors.

"These authors contributed equally to this work.

Correspondence to: Sook-Hyang Jeong, MD, PhD. Department of Internal Medicine, Seoul National University Bundang Hospital, College of Medicine, Seoul National University, 82 Gumi-ro, 173 Beon-gil, Bundang-gu, Seongnam 13620, Republic of Korea. Email: jsh@snubh.org.

Background: This study investigated the association of 3 components of body composition (sarcopenia, intramuscular fat deposition and visceral adiposity) with the overall or recurrence-free survival of hepatocellular carcinoma (HCC) patients who underwent curative hepatic resection.

Methods: One hundred sixty newly diagnosed and surgically treated HCC patients were retrospectively enrolled from 2003 to 2011. Three items of body composition were measured using the $3^{\text {rd }}$ lumbar level image of preoperative computed tomography (CT): psoas muscle index (PMI), psoas muscle attenuation (PMA), and visceral adipose tissue index (VATI). Sex-specific optimal cut-off for each item was determined from receiver-operating characteristic curves.

Results: The HCC patients showed a median age of 55 years, $75 \%$ of male, $78 \%$ of hepatitis B surface antigen positivity, and $96 \%$ of Child-Pugh A. The sarcopenic group (PMI less than the sex-specific cutoff of $3.33 \mathrm{~cm}^{2} / \mathrm{m}^{2}$ for men and $2.38 \mathrm{~cm}^{2} / \mathrm{m}^{2}$ for women) had $17.5 \%$ of the patients with a lower PMA (more fat deposition) but similar VATI compared to the non-sarcopenic group. PMI showed a positive correlation with PMA ( $\rho=0.493, \mathrm{P}<0.001$ ), while there was no significant correlation between PMI and VATI, and between PMA and VATI. On the multivariate analysis, a high PMI and low VATI were independent factors affecting overall survival while PMA was not. Nevertheless, PMI and VATI were not independent factors for recurrence-free survival.

Conclusions: In curatively resected HCC patients, sarcopenia and high visceral adiposity predict poor overall survival but not recurrence-free survival, while PMA did not predict overall survival.

Keywords: Sarcopenia; myosteatosis; visceral adiposity; hepatocellular carcinoma (HCC); survival

^ ORCID: Hee Yoon Jang, 0000-0001-7709-8673; Gwang Hyeon Choi, 0000-0002-8795-8427; Sung Ho Hwang, 0000-0001-5356-1993; Eun Sun Jang, 0000-0003-4274-2582; Jin-Wook Kim, 0000-0003-0934-3344; Jung Wha Chung, 0000-0003-3648-3406; Sook-Hyang Jeong, 0000-00024916-7990. 
Submitted Oct 02, 2020. Accepted for publication Nov 27, 2020.

doi: 10.21037/tcr-20-2974

View this article at: http://dx.doi.org/10.21037/tcr-20-2974

\section{Introduction}

Liver cancer is the fifth most common cancer and the third leading cause of cancer-related deaths worldwide in 2018 estimated by the World Health Organization (1), and the 5 -year survival rate of hepatocellular carcinoma (HCC) was reported as $18.4-40.4 \%(2,3)$. While the performance status of HCC patients, tumor extent, and underlying liver function are major factors that determine clinical outcomes, recent studies reported that the indices of body composition including sarcopenia and regional fat distribution also affect the overall survival of HCC. Sarcopenia was consistently associated with a poor overall survival in variously treated patients with HCC (4-10), but its association with recurrence-free survival was controversial $(7,8,10-13)$. Moreover, intramuscular fat (IMF) deposition and visceral adiposity may independently predict mortality in patients with HCC, as shown in previous studies $(14,15)$.

However, studies on the relationship between the components of body composition and HCC are limited in Asian regions where hepatitis $\mathrm{B}$ virus (HBV) infection is the major cause of HCC. HCC patients with HBV were younger by about 10 years on average compared with those with hepatitis $\mathrm{C}$ virus ( $\mathrm{HCV}$ ) infection, suggesting a different profile of sarcopenia according to the etiology of HCC and the regions (16). The prevalence and characteristics of sarcopenia among HCC patients are not clear, and only one previous study was done in Korea (6). Moreover, the association between IMF deposition or visceral adiposity with the overall survival needs to be studied.

Using the pretreatment computed tomography (CT) image, psoas muscle index (PMI) as a validated marker of sarcopenia, psoas muscle attenuation (PMA) as a marker of IMF deposition (myosteatosis), and visceral adipose tissue index (VATI) as a marker of visceral adiposity, can be measured in HCC patients. This study investigated the association between each of the 3 components of the body composition (PMI, PMA and VATI) and the overall or recurrence-free survival of HCC patients who underwent curative hepatic resection. We present the following study in accordance with the REMARK reporting checklist (available at http://dx.doi. org/10.21037/tcr-20-2974).

\section{Methods}

\section{Patients, clinical data collection, and follow up}

Between May 2003 and December 2011, 182 HCC patients who underwent curative hepatic resection were retrospectively enrolled in this study at Seoul National University Bundang Hospital. Among them, 22 patients were excluded because their preoperative CT images were not available or inappropriate to measure the body components. Therefore, 160 patients with pathologically proven HCC were the final subjects of this study. Preoperative CT images taken within 60 days before surgery were considered to be appropriate. The study was conducted in accordance with the Declaration of Helsinki (as revised in 2013). This study was approved by the Institutional Review Board of Seoul National University Bundang Hospital (B-1603-340-111), and informed consent was waived due to the retrospective nature of this study.

Clinical and pathological data were collected from electronic medical records, which included gender, height, weight, etiology of HCC, alcohol intake and smoking history, comorbidities, blood levels of alpha-fetoprotein (AFP), albumin, serum aspartate aminotransferase (AST), alanine aminotransferase (ALT), total bilirubin, prothrombin time (PT) expressed as the international normalized ratio (INR), platelet count, creatinine, and sodium, Child-Pugh score and class, model for end stage liver disease-sodium (MELD-Na) score, tumor size, tumor number, Tumor-Node-Metastasis (TNM) stage based on American Joint Committee on Cancer, 8th edition (17), and Barcelona Clinic Liver Cancer (BCLC) stage.

The patients were followed until October 2019 for a median duration of 5.9 years. Overall survival was the time from HCC diagnosis until death due to any cause and recurfree survival was defined as the time from HCC diagnosis until first recurrence (loco-regional or distant metastasis) or death due to any cause, whichever was observed first. The mortality of each patient was confirmed by requested data from Statistics Korea, and cause of death was checked by review of the medical records. Recurrence of HCC was confirmed by medical records and CT or magnetic resonance imaging (MRI) images. 


\section{Measurement of the body composition variables and determination of their cutoffs}

Contrast-enhanced CT examination was performed with a multidetector-row CT machine (Brilliance 64 or iCT256; Philips Medical Systems, Cleveland, OH, USA). Intravenous non-ionic contrast material $(2 \mathrm{~mL} / \mathrm{kg}$, iomeprol; $350 \mathrm{mg}$ iodine/mL, Iomeron 350; Bracco, Milano, Italy) was administered through the antecubital vein using a power injector (Stellant D, Medrad, Indianola, PA, USA).

The 2-dimensional cross-sectional area of the skeletal muscle including both psoas muscles and adipose tissue area were measured at the middle level of the third lumbar vertebra (L3) where both pedicles were clearly visible on the pre-enhanced preoperative CT scans. Using the Rapidia 3D software (v2.8; INFINITT Healthcare, Seoul, Korea), the area of the bilateral psoas muscles was manually outlined in the setting from 31 to 100 Hounsfield units (HUs) to exclude strictly the vasculature and areas of fatty infiltration. The measured bilateral psoas muscle area was divided by the patient height squared for the calculation of the PMI $\left(\mathrm{cm}^{2} / \mathrm{m}^{2}\right)(5,18-20)$.

Using the same CT images, mean PMA was calculated to assess psoas muscle fat deposition (14). A low PMA indicates increased IMF content that contributes to muscle weakness independent of the age-associated loss of muscle mass (14). The visceral adipose tissue area was measured in a $\mathrm{HU}$ setting from -150 to -50 , and visceral adipose tissue area index $\left(\mathrm{cm}^{2} / \mathrm{m}^{2}\right)$ was calculated by dividing by the patient height squared for precise measurements, two trained researchers, blinded to all the clinical data, independently measured the same group of CT images from 20 randomly selected patients and compared the results. The interobserver agreement was $97 \%$, and then, one researcher measured the whole series of CT images from the subjects. The researcher who was measured these parameters was not involved in statistical analysis.

To determine the sex-specific cut-offs for PMI, PMA, and VATI, optimum stratification by the R software package (version 3.3.2; R Foundation for statistical Computing, Vienna, Austria; http://www.R-project.org/) was performed, which found the most significant value by using the maximal log-rank statistics (21). This method has been described previously in the literature as a method to solve the threshold value of a continuous co-variate such as the PMI (22).

\section{Statistical analysis}

Continuous variables are presented as the medians with interquartile ranges (IQRs), unless otherwise indicated. Categorical variables are presented as numbers and percentages. Differences between the two groups were analyzed using Mann-Whitney test for continuous variables and Fisher's exact test for categorical variables. Spearman's rank correlation was used to find the correlation between the PMI and either of the PMA or VATI, and the coefficient of correlation was indicated as Spearman's rho $(\rho)$.

Overall survival and recurrence-free survival were analyzed with Kaplan-Meier curves, and the log-rank test was used to compare the survival between the two groups. A $\mathrm{P}$ value $<0.05$ was considered statistically significant. To confirm the prognostic role of PMI, PMA, and VATI in the overall and recurrence-free survival, univariate and multivariate analyses were performed. The variables showing a $\mathrm{P}$ value $<0.1$ in the univariate analysis were included in the multivariate Cox proportional hazards model, and hazard ratios (HRs) with 95\% confidence intervals (CIs) were computed. The data were analyzed with SPSS (version 22.0; SPSS Inc., Chicago, IL, USA).

\section{Results}

\section{Preoperative clinical characteristics and survival of HCC patients with comparison between the sarcopenic and non- sarcopenic group}

The clinical and pathological characteristics of the 160 patients are shown in Table 1 with a comparison between the sarcopenic and non-sarcopenic group according to the sex-specific PMI cutoff of $3.33 \mathrm{~cm}^{2} / \mathrm{m}^{2}$ for men and $2.38 \mathrm{~cm}^{2} / \mathrm{m}^{2}$ for women. Overall, the subjects had a mean age of 55 years, $75 \%$ were males, and $78.2 \%$ had a hepatitis B surface antigen (HBsAg) positivity. Additionally, 96.3\% had good liver function indicated by the Child-Pugh class A score. The mean diameter of HCC was $4.1 \mathrm{~cm}$, and $74 \%$ had a BCLC stage 0 or A. There was no missing value in variables.

During a median follow-up of 7.9 years, 72 patients (45.0\%) died, and 89 patients $(55.6 \%)$ showed HCC recurrence. The cause of death was progression of HCC $(n=41)$, cirrhosis-related hepatic dysfunction $(n=13)$, postoperative hepatic dysfunction $(\mathrm{n}=2)$, non-liver related cause $(n=11)$, and unknown cause $(n=5)$. The median overall survival was 7.9 years (range, $0.1-15.2$ years), and the median recurrence-free survival was 3.2 years (range, $0.1-15.2$ years).

According to the above sex-specific cutoff, the prevalence 
Table 1 Preoperative clinical characteristics and body compositions of 160 HCC patients with comparison between sarcopenic and nonsarcopenic group

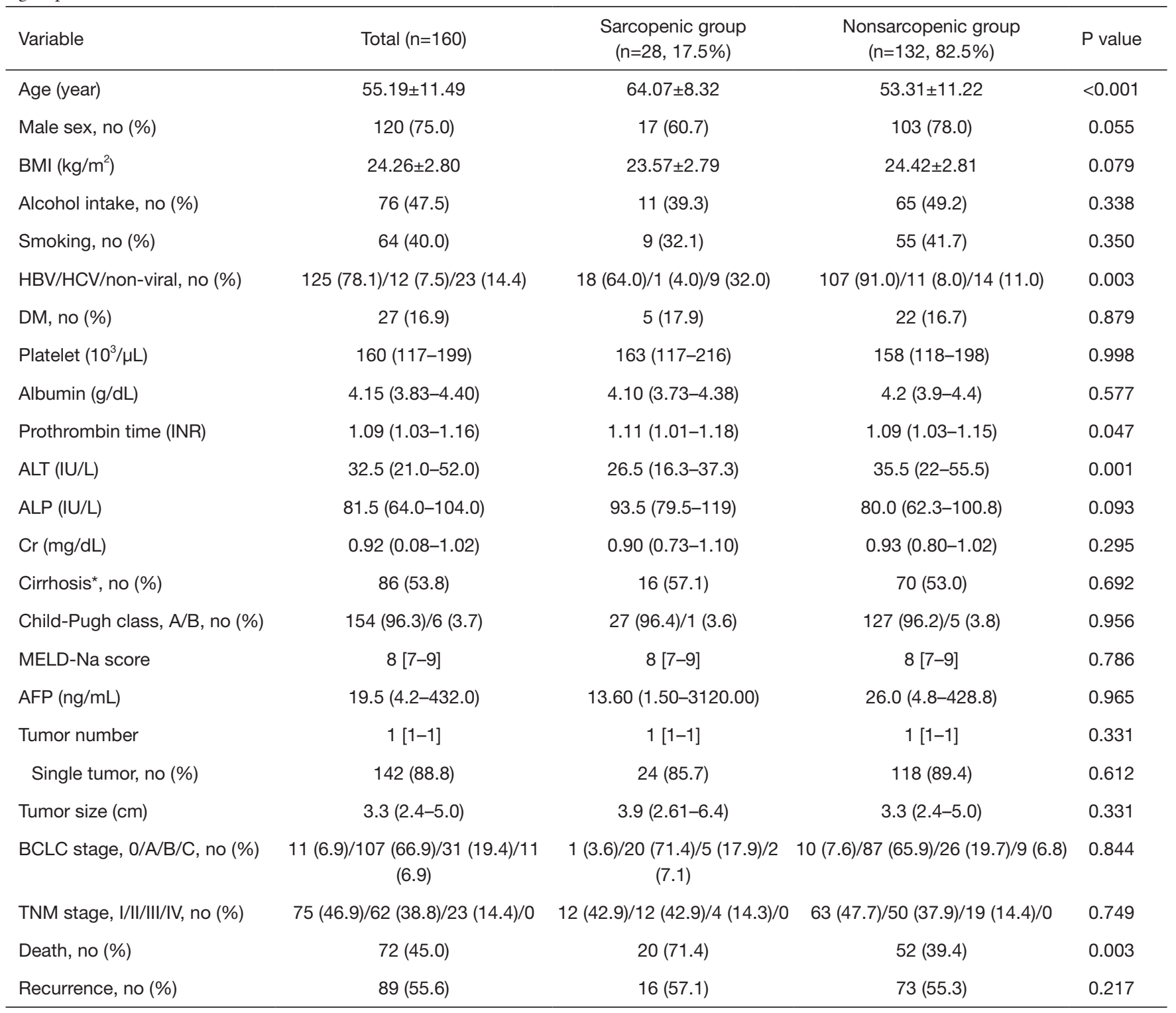

${ }^{*}$, cirrhosis was pathologically confirmed. HCC, hepatocellular carcinoma; BMI, body mass index; HBV, hepatitis B virus; HCV, hepatitis C virus; DM, diabetes mellitus; INR, international normalized ratio; ALT, alanine aminotransferase; ALP, alkaline phosphatase; Cr, creatinine; MELD, model for end-stage liver disease; AFP, alpha-fetoprotein; BCLC, Barcelona Clinic Liver Cancer; TNM, tumor-node-metastasis.

of sarcopenia was $17.5 \%$. The sarcopenic group had a higher age, higher proportion of non-viral etiology, and lower ALT level. Moreover, the 5-year overall survival rate in the sarcopenic group (53.6\%) was significantly lower than in non-sarcopenic group $(75.3 \%)$, while recurrencefree survival was not different between the two groups.

The cumulative curve of overall and recurrence-free survival between the sarcopenic and non-sarcopenic group is shown in Figures 1 and 2, respectively. The sarcopenic group showed significantly shorter overall survival after surgical resection than the non-sarcopenic group (HR, 2.404; 95\% CI, 1.433-4.034; $\mathrm{P}=0.001$ ). The sarcopenic group showed shorter recurrence free survival than the nonsarcopenic group, however it was not statistically significant 


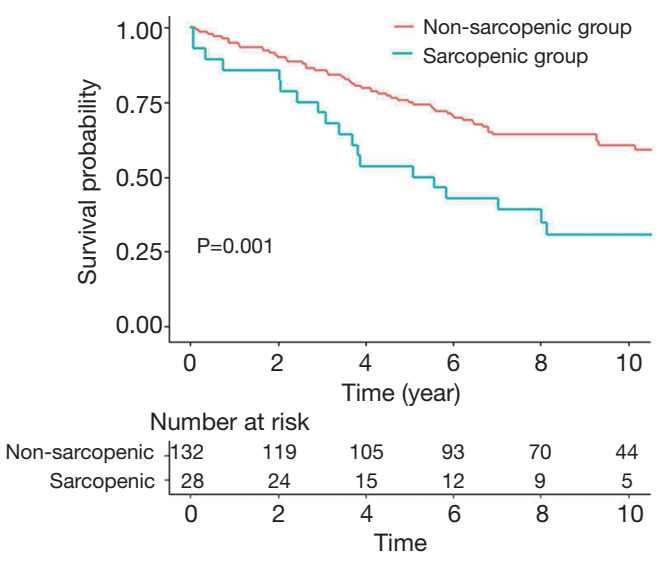

Figure 1 Overall survival of HCC patients according to the presence of sarcopenia. Among the 160 surgically treated HCC patients, the prevalence of sarcopenia defined as a low PMI (PMI less than the sex-specific cutoff of $3.33 \mathrm{~cm}^{2} / \mathrm{m}^{2}$ for men and $2.38 \mathrm{~cm}^{2} / \mathrm{m}^{2}$ for women) was $17.5 \%$. The sarcopenic group showed a significantly lower overall survival than the non-sarcopenic group. HCC, hepatocellular carcinoma; PMI, psoas muscle index.

(HR, 1.477; 95\% CI, 0.924-2.352; $\mathrm{P}=0.103)$.

\section{Levels of PMI, P MA, and VATI with their optimal cutoffs and their correlations in the HCC patients}

The median values of the PMI, PMA, and VATI in HCC patients are shown in Table 2 with a comparison between the sarcopenic and nonsarcopenic group. The median PMI of the overall patients was $4.14 \mathrm{~cm}^{2} / \mathrm{m}^{2}\left(4.55 \mathrm{~cm}^{2} / \mathrm{m}^{2}\right.$ for men and $2.84 \mathrm{~cm}^{2} / \mathrm{m}^{2}$ for women, respectively) and the median PMA was $49.90 \mathrm{HU}$ (50.56 HU for men and $46.76 \mathrm{HU}$ for women, respectively). The median VATI $37.70 \mathrm{~cm}^{2} / \mathrm{m}^{2}$ $\left(40.14 \mathrm{~cm}^{2} / \mathrm{m}^{2}\right.$ for men and $26.81 \mathrm{~cm}^{2} / \mathrm{m}^{2}$ for women, respectively).

The sex-specific cut-offs for PMI ascertained by optimum stratification were $3.33 \mathrm{~cm}^{2} / \mathrm{m}^{2}$ for men and $2.38 \mathrm{~cm}^{2} / \mathrm{m}^{2}$ for women. The sex-specific cut-offs for PMA were $46.08 \mathrm{HU}$ for men and $48.42 \mathrm{HU}$ for women. The sex-specific cut-offs for VATI were $30.39 \mathrm{~cm}^{2} / \mathrm{m}^{2}$ for men and $44.70 \mathrm{~cm}^{2} / \mathrm{m}^{2}$ for women. The patients below the above-mentioned cutoffs for the PMI, PMA, and VATI were classified as the sarcopenic group, low PMA group, and low VATI group, respectively.

In the correlation analysis, the PMI showed a positive correlation with PMA $(\rho=0.50, \mathrm{P}<0.001$, Figure $3 A)$, it means that patients with sarcopenia tend to have more

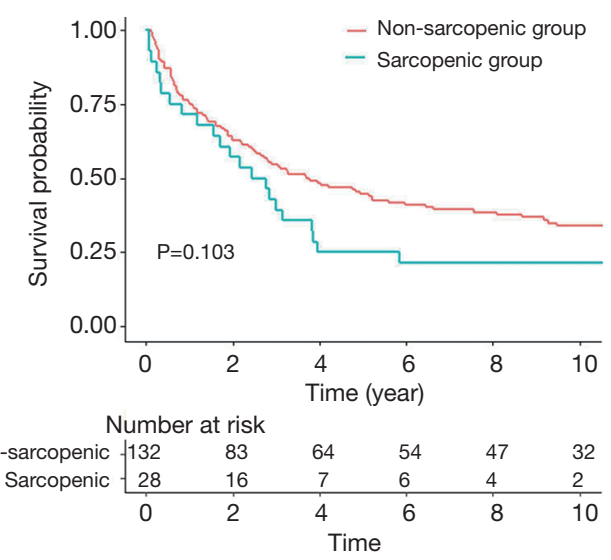

Figure 2 Recurrence free survival of HCC patients according to the presence of sarcopenia. Among the 160 surgically treated HCC patients, recurrence-free survival was not significantly different between the sarcopenic and the non-sarcopenic group. Sarcopenia was defined as a low PMI (PMI less than the sex-specific cutoff of $3.33 \mathrm{~cm}^{2} / \mathrm{m}^{2}$ for men and $2.38 \mathrm{~cm}^{2} / \mathrm{m}^{2}$ for women. HCC, hepatocellular carcinoma; PMI, psoas muscle index

myosteatosis and vice versa. However, there is no significant correlation between PMI and VATI $(\rho=0.05, \mathrm{P}=0.52$, Figure $3 B$ ), it means that sarcopenia and visceral obesity are independent variables. The correlation profiles of each body composition item and demographic or functional variables are shown in the heat map (Figure 4).

\section{The effect of PMI, PMA, and VATI on the overall survival or recurrence-free survival in HCC patients}

The univariate analysis showed that an age 65 years or older (HR, 2.167; 95\% CI, 1.331-3.526; $\mathrm{P}=0.002$ ), platelet count less than $100 \times 10^{3} / \mathrm{mL}$ (HR, 2.531; 95\% CI, $1.478-$ 4.332; $\mathrm{P}=0.001$ ), MELD-Na score 8 or more (HR, 2.087; 95\% CI, 1.283-3.395; P=0.003), TNM stage II + III (HR, 2.376; 95\% CI, 1.452-3.887; P=0.001), low PMA (HR, 1.754; 95\% CI, 1.046-2.941; $\mathrm{P}=0.033$ ), and low PMI (HR, 2.404; 95\% CI, 1.433-4.034; $\mathrm{P}=0.001$ ) were significant factors associated with the overall survival of the HCC patients after surgical resection (Table 3) and high VATI (HR, 1.600; 95\% CI, 0.985-2.600; $\mathrm{P}=0.058$ ) showed marginally associated with overall survival. In the multivariate Cox proportional hazard analysis, a platelet count less than $100 \times 10^{3} / \mathrm{mL}$ (HR, 3.073; 95\% CI, 1.726-5.472; $\mathrm{P}<0.001$ ), MELD-Na score 8 or more (HR, 1.868; 95\% CI, 1.127-3.096; P=0.015), TNM stage II + III 
Table 2 Preoperative characteristics of body compositions of 160 HCC patients with comparison between sarcopenic and nonsarcopenic group

\begin{tabular}{|c|c|c|c|c|}
\hline Body composition variables & Total $(n=160)$ & $\begin{array}{c}\text { Sarcopenic group } \\
(n=28,17.5 \%)\end{array}$ & $\begin{array}{c}\text { Nonsarcopenic group } \\
(n=132,82.5 \%)\end{array}$ & $P$ value \\
\hline Age (year) & $55.19 \pm 11.49$ & $64.07 \pm 8.32$ & $53.31 \pm 11.22$ & $<0.001$ \\
\hline $\operatorname{BMI}\left(\mathrm{kg} / \mathrm{m}^{2}\right)$ & $24.26 \pm 2.80$ & $23.57 \pm 2.79$ & $24.42 \pm 2.81$ & 0.079 \\
\hline Psoas muscle area $\left(\mathrm{cm}^{2}\right)$ & $11.29(8.06-14.72)$ & $5.31(4.10-7.75)$ & $12.06(10.09-16.26)$ & $<0.001$ \\
\hline Male & $12.47(10.34-16.92)$ & $7.40(8.20-5.97)$ & $13.37(11.31-17.40)$ & \\
\hline Psoas muscle index $\left(\mathrm{cm}^{2} / \mathrm{m}^{2}\right)$ & $4.14(3.24-5.05)$ & $2.21(1.74-2.81)$ & $4.35(3.78-5.48)$ & $<0.001$ \\
\hline Female & $2.84(2.27-3.86)$ & $1.74(1.63-2.17)$ & $3.31(2.69-3.99)$ & \\
\hline Male & $50.56(47.83-53.32)$ & $46.76(10.98-48.67)$ & $50.98(48.36-53.72)$ & \\
\hline Visceral adipose tissue area $\left(\mathrm{cm}^{2}\right)$ & $105.05(57.08-151.16)$ & $118.41(81.58-167.54)$ & $97.96(53.96-150.08)$ & 0.850 \\
\hline Female & 64.69 (38.92-104.92) & $112.67(20.75-166.52)$ & $63.80(42.53-83.94)$ & \\
\hline Male & $117.72(73.72-168.00)$ & $124.17(102.05-186.99)$ & $116.26(65.89-168.04)$ & \\
\hline Visceral adipose tissue index $\left(\mathrm{cm}^{2} / \mathrm{m}^{2}\right)$ & $37.70(21.94-57.64)$ & $45.81(30.92-68.95)$ & $36.05(20.97-56.26)$ & 0.766 \\
\hline Female & $26.81(16.52-43.54)$ & $45.71(8.21-71.13)$ & $25.65(18.78-35.86)$ & \\
\hline Male & $40.14(25.68-60.46)$ & $45.90(33.67-69.29)$ & $38.27(22.87-60.25)$ & \\
\hline
\end{tabular}

HCC, hepatocellular carcinoma; BMI, body mass index; HU, Hounsfield unit.
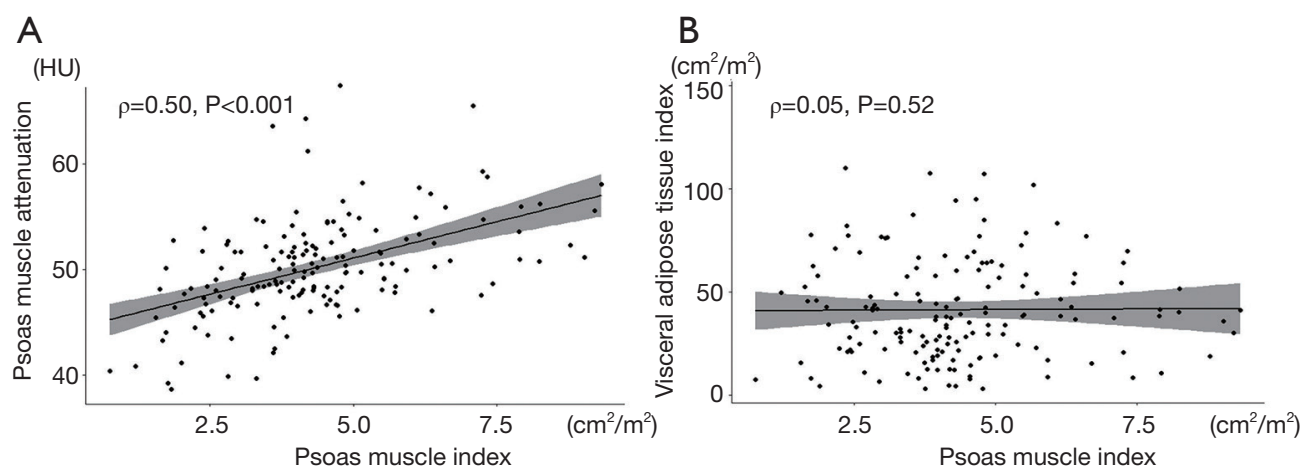

Figure 3 Correlations between the psoas muscle index (PMI) and psoas muscle attenuation (PMA), and PMI and visceral to subcutaneous adipose tissue ratio (VATI). Among the body composition items, PMI showed a positive correlation with $\mathrm{PMA}(\rho=0.493$, $\mathrm{P}<0.001$ ) suggesting that a higher muscle mass was correlated with less intramuscular fat deposition (A). However, there was no significant correlation between the PMI and VATI, suggesting that muscle mass was not related with visceral adiposity, in the 160 curatively resected HCC patients (B). HCC, hepatocellular carcinoma. 


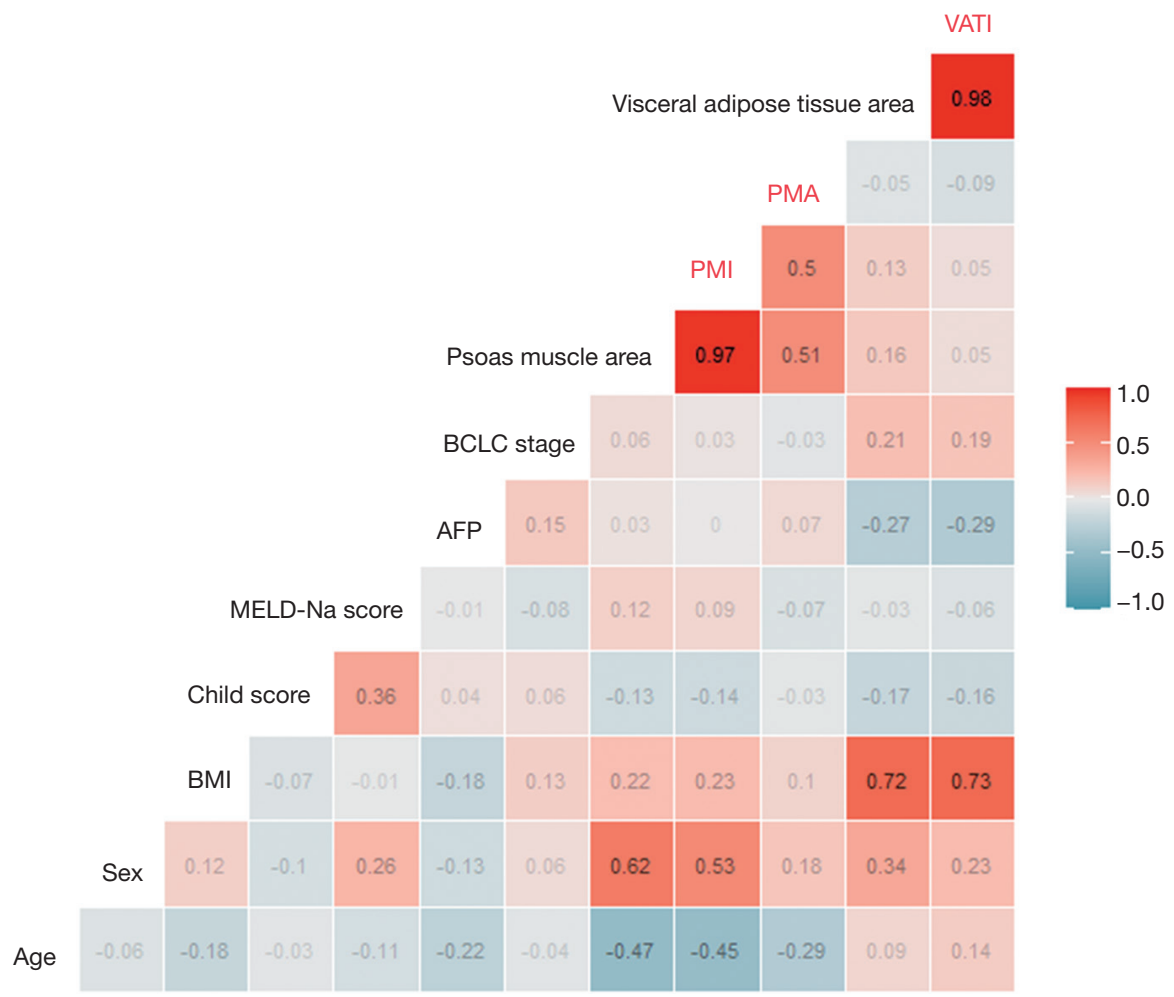

Figure 4 Correlation plot including demographics, liver function, tumor characteristics, and the components of body compositions. The heat map showing a red background indicates a positive correlation, the blue background indicates negative correlation, and the numbers in the box indicate rho $(\rho)$. PMI positively correlates with the psoas muscle area $(\rho=0.97, \mathrm{P}<0.001), \mathrm{PMA}(\rho=0.50, \mathrm{P}<0.001)$, male sex $(\rho=0.53$, $\mathrm{P}<0.001)$, and $\mathrm{BMI}(\rho=0.23, \mathrm{P}=0.003)$, whereas it negatively correlates with age $(\rho=-0.45, \mathrm{P}<0.001)$. The PMA positively correlate with the psoas muscle area $(\rho=0.51 \mathrm{P}<0.001)$, and male sex $(\rho=0.18 \mathrm{P}=0.03)$, whereas it negatively correlates with age $(\rho=-0.29$, $\mathrm{P}<0.001)$. The VATI positively correlates with the visceral tissue area $(\rho=0.98, P<0.001)$, BMI $(\rho=0.73, P<0.001)$, and male sex $(\rho=0.23, P=0.003)$ and negatively correlation with the Child-Pugh score $(\rho=-0.16, \mathrm{P}=0.045)$ and AFP $(\rho=-0.29, \mathrm{P}<0.001)$. AFP, alpha-fetoprotein; BMI, body mass index; MELD, model for end-stage liver disease; PMI, psoas muscle index; PMA, psoas muscle attenuation; VATI, visceral adipose tissue index.

(HR, 2.466; 95\% CI, 1.484-4.095; $\mathrm{P}<0.001$ ), high VATI (HR, 1.770; 95\% CI, 1.036-3.021; $\mathrm{P}=0.037$ ), and low PMI (HR, 2.245; 95\% CI, 1.021-4.937; $\mathrm{P}=0.044)$ remained independent factors associated with the overall survival.

In terms of the recurrence-free survival, a low platelet count (HR, 2.132; 95\% CI, 1.341-3.389; $\mathrm{P}=0.001$ ), high MELD-Na score (HR, 1.847; 95\% CI, 1.258-2.711; $\mathrm{P}=0.002)$, and TNM stage II + III (HR, 1.734; 95\% CI, 1.186-2.536; $\mathrm{P}=0.005)$ showed a significant association, whereas, the PMI, PMA, and VATI did not in the univariate Cox proportional hazard analysis. In the multivariate Cox proportional hazard analysis, a low platelet count (HR, 2.174; 95\% CI, 1.354-3.491; $\mathrm{P}=0.001)$, high MELD-Na score (HR, 1.566; 95\% CI, 1.055-2.324; $\mathrm{P}=0.026$ ), and TNM stage II + III (HR, 1.739; 95\% CI, 1.182-2.556; $\mathrm{P}=0.005)$ remained independent factors associated with the recurrence-free survival (Table 4).

Therefore, the presence of the PMI and VATI were independent factors for the overall survival. However, neither the PMI, nor the VATI was an independent factor for the recurrence-free survival.

\section{Discussion}

In this study, 3 components of body composition (sarcopenia, IMF deposition and visceral adiposity) were investigated among 160 Korean HCC patients with mostly HBV related cause and with good liver function who underwent curative resection. The prevalence of sarcopenia defined as a low PMI according to a sex-specific cutoff was $17.5 \%$, and there was a significant positive correlation between the PMI and PMA, but no correlation between 
Table 3 Factors associated with overall mortality according to Cox Proportional Hazard model in the patients who underwent curative hepatic resection for HCC

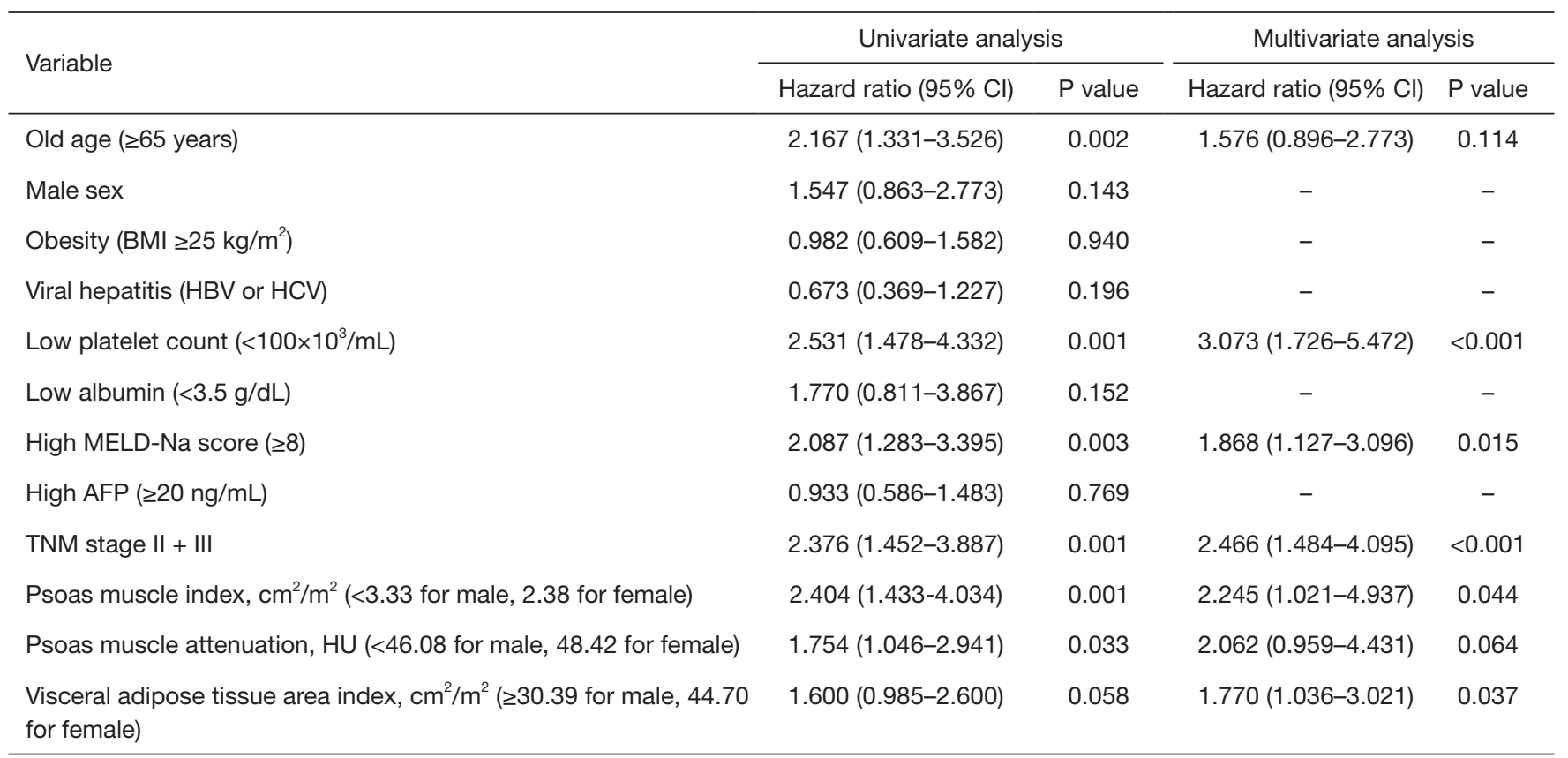

HCC, hepatocellular carcinoma; $\mathrm{Cl}$, confidence interval; BMI, body mass index; HBV, hepatitis B virus; HCV, hepatitis C virus; MELD, model for end-stage liver disease; AFP, alpha-fetoprotein; TNM, tumor-node-metastasis; HU, Hounsfield unit.

Table 4 Factors negatively associated with recurrence-free survival according to Cox Proportional Hazard model in the patients who underwent curative hepatic resection for HCC

\begin{tabular}{|c|c|c|c|c|}
\hline Variable & \multicolumn{2}{|c|}{ Univariate analysis } & \multicolumn{2}{|c|}{ Multivariate analysis } \\
\hline Old age ( $\geq 65$ years) & $1.115(0.722-1.722)$ & 0.623 & - & - \\
\hline Male sex & $1.496(0.945-2.370)$ & 0.086 & $1.355(0.849-2.163)$ & 0.203 \\
\hline Obesity (BMI $\left.\geq 25 \mathrm{~kg} / \mathrm{m}^{2}\right)$ & $0.905(0.613-1.335)$ & 0.614 & - & - \\
\hline Low platelet count $\left(<100 \times 10^{3} / \mathrm{mL}\right)$ & $2.132(1.341-3.389)$ & 0.001 & $2.174(1.354-3.491)$ & 0.001 \\
\hline Low albumin $(<3.5 \mathrm{~g} / \mathrm{dL})$ & $1.203(0.585-2.471)$ & 0.616 & - & - \\
\hline High MELD-Na score $(\geq 8)$ & $1.847(1.258-2.711)$ & 0.002 & $1.566(1.055-2.324)$ & 0.026 \\
\hline High AFP ( $\geq 20$ ng/mL) & $0.998(0.686-1.452)$ & 0.992 & - & - \\
\hline Psoas muscle attenuation, $\mathrm{HU}$ (<46.08 for male, 48.42 for female) & $1.253(0.801-1.959)$ & 0.323 & - & - \\
\hline $\begin{array}{l}\text { Visceral adipose tissue area index, } \mathrm{cm}^{2} / \mathrm{m}^{2} \text { ( } \geq 30.39 \text { for male, } 44.70 \\
\text { for female) }\end{array}$ & $0.726(0.495-1.063)$ & 0.100 & - & - \\
\hline
\end{tabular}

HCC, hepatocellular carcinoma; Cl, confidence interval; BMI, body mass index; HBV, hepatitis B virus; HCV, hepatitis C virus; MELD, model for end-stage liver disease; AFP, alpha-fetoprotein; TNM, tumor-node-metastasis; HU, Hounsfield unit. 
PMI and VATI. A high PMI and low VATI according to sex-specific cutoff were independent factors predicting overall survival, but both were not independent factors to predict recurrence-free survival in the multivariate analysis.

CT provides a validated tool for measurement of body composition in patients with various cancers including HCC, by quantification of area and attenuation of psoas muscle and visceral fat in the abdomen. Low muscle area according to a sex-specific cutoff is a main component of sarcopenia while it does not represent muscle strength, and the prevalence of sarcopenia in this study was $17.5 \%$. The sarcopenic prevalence in HCC patients has been reported as a wide range from $10 \%$ to $54 \%$ according to the different characteristics of study subjects including age, sex, tumor stage, liver function, and treatment modality $(7,10,13,14)$. Our subjects were relatively young (median age of 55 years) and had good liver function and a regional stage of the tumor as indications of curative resection, so that the sarcopenic prevalence was low, comparable to other studies including patients who underwent curative resection.

The sarcopenic group in this study had an older age, higher proportion of non-viral cause, higher PT, but a lower ALT level compared with the non-sarcopenic group. However, tumor stage and tumor aggressiveness in terms of the alpha fetoprotein level, and functional liver status in terms of the MELD-Na score were not different between the two groups. Therefore, the presence of sarcopenia seems to be dominantly related to the patient's age and comorbidities suggested by non-viral causes including alcohol and nonalcoholic fatty liver diseases. The mechanisms of accelerated sarcopenia in patients with liver cirrhosis include frequent protein-energy malnutrition, decreased branched chain amino acid (BCAA) levels in the blood and muscles, elevated levels of ammonia with subsequent myostatin expression, and elevated levels of reactive oxygen species and inflammatory cytokines, all of which lead to a negative protein balance (23). In the cancer cachexia animal model, the activated ubiquitin-proteasome system and activated $\mathrm{NF}-\kappa \mathrm{B}$ signaling were implicated (24), however, the specific mechanism underlying the association of sarcopenia in HCC rather than in cirrhosis needs further study.

Sarcopenia is a well-known predictor for HCC survival as confirmed in this study $(4-10,12)$; the low PMI group showed a significantly lower overall survival compared to the that of high PMI group, and a low PMI was an independent factor for overall survival in the multivariate analysis. According to a recent meta-analysis including 13 related studies, sarcopenia was consistently associated with a lower overall survival (adjusted HR 1.95, 95\% CI, 1.60-2.37) regardless of the characteristics of the subjects and treatment modalities such as resection, transarterial chemoembolization, intraarterial chemotherapy, targeted therapy, and liver transplantation (11). However, recurrence-free survival was not significantly different between the sarcopenic and non-sarcopenic group in this study although the actual recurrence rate showed a higher tendency in the sarcopenic group. There have been controversial results about the effect of sarcopenia on the tumor recurrence $(7,8,10-13)$. In one study, sarcopenic patients tended to have undifferentiated HCCs and more satellite nodules suggesting tumor aggressiveness than that of the nonsarcopenic patients (10). However, our data did not support such a difference in the tumor factors according to sarcopenia. Therefore, whether sarcopenia affects HCC recurrence after surgical resection needs further study. Sarcopenia is important because it affects not only the overall survival but also the patient's quality of life. As seen in a Japanese study, supplementation of dietary BCAA showed beneficial effects on the prevention of sarcopenia progression in patients with liver cirrhosis (25). Therefore, an evaluation of sarcopenia followed by an intervention for sarcopenia by way of nutritional support and exercise reinforcement for HCC patients may improve the prognosis.

The cutoff value defining sarcopenia varies widely by age, sex, ethnicity and/or region. In Korea, a national representative study based on the $4^{\text {th }}$ Korean National Health and Nutritional Examination Surveys (KNHNES) evaluated the height-adjusted appendicular skeletal muscle mass (ASM) of a young healthy adult population using dualenergy X-ray absorptiometry (DEXA) and reported that the determined cutoff values of sarcopenia for Korean men and women were 6.58 and $4.59 \mathrm{~kg} / \mathrm{m}^{2}$, respectively (26). Based on the linear correlation between the psoas muscle area and appendicular skeletal muscle by DEXA, a regression equation was produced (20). In another Korean study including 178 variously treated HCC patients, the authors determined the sex-specific cutoffs for the L3 skeletal muscle index using the receiver operating characteristic (ROC) curve, which were 45.8 for men and $43.0 \mathrm{~cm}^{2} / \mathrm{m}^{2}$ for women (6). Therefore, even in Korea, the cutoff level for defining sarcopenia varies, which needs to be standardized in the future. Our study measured psoas muscle area rather than whole skeletal muscle area in the L3 level. It could not directly compare with the study using ASM. Because no consensus value for CT-based sarcopenia presented by the sex-specific PMI has 
been reported in Korean populations, we determined the cutoff value associated with a poor survival in HCC patients in this study using the ROC curve $\left(3.33 \mathrm{~cm}^{2} / \mathrm{m}^{2}\right.$ for men and $2.38 \mathrm{~cm}^{2} / \mathrm{m}^{2}$ for women, respectively), which were lower than those in U.S. (7.840 and $6.421 \mathrm{~cm}^{2} / \mathrm{m}^{2}$ for men and women, respectively) (27), but were relatively close to those in Japan (4.24 and $2.50 \mathrm{~cm}^{2} / \mathrm{m}^{2}$ for men and women) (19).

The role of myosteatosis-ectopic fat deposition in muscle in the prognosis of HCC has not been studied much. A low muscle attenuation on CT indicates IMF deposition, which contributes to muscle weakness independent of the age-associated loss in muscle mass (28). In this study, myosteatosis indicated as the PMA was significantly correlated with the PMI, suggesting a high muscle mass tends to have less fat. Moreover, the PMA was significantly associated with overall survival in the univariate analysis, but it did not remain in the multivariate analysis. It may be related to the low proportion of sarcopenic patients in this study, which may lessen the independent effect of PMA on the survival due to the low statistical power. Hamaguchi et al. reported that preoperative muscle steatosis evaluated by intramuscular adipose tissue content (IMAC) was significantly correlated with the incidence of postoperative major and infectious complications in patients undergoing hepatectomy for HCC $(15,29)$. Fujiwara et al. showed that IMF deposition evaluated by mean muscle attenuation was a significant predictor of all-cause and liver-related mortality independent of cancer stage or liver function in 1,490 variously treated HCC patients (14). The biological basis of this predictive value of muscle attenuation for survival is unknown (30), but there is direct evidence that the muscle biopsy material of cancer patients develops increased numbers of intramyocellular lipid droplets seen in electron microscopy, which are more abundant in the muscles of patients experiencing progressive cancer-associated weight loss compared to weight stable cancer patients. Moreover, the composition of the lipid components in the muscles may be an important in driving the pathology of the muscles. For example, accumulation of diacylglycerols, but not triacylglycerol, is associated with insulin resistance in nonadipose tissues. The molecular mechanism contributing to myosteatosis in HCC awaits further investigation. In addition, sarcopenia and IMF deposition are associated with insulin resistance (31), vitamin D deficiency (32), and increased inflammatory cytokine levels, such as interleukin-6 (IL-6) (33), all of which are associated with the progression of liver fibrosis and HCC $(34,35)$.

A high VATI is an independent factor for predicting overall survival in our study. It is compatible with the results of previous studies. A high visceral adipose area was associated with the poor survival of advanced HCC patients treated with sorafenib and brivanib in France, suggesting that visceral fat may be related to resistance to anti-angiogenic drugs (36). Moreover, Fujiwara et al. showed that the visceral distribution of the fat rather than the amount of the fat determines a poor prognosis for HCC patients, probably by way of increasing the proinflammatory adipokines and impaired suppression of free fatty acid release in response to insulin (14). Hamaguchi et al. also reported that the visceral to subcutaneous adipose ratio, skeletal muscle index, and IMAC were independent factors for mortality after hepatectomy in HCC patients (15).

Visceral adipocytes mainly secret proinflammatory cytokines such as tumor necrosis factor- $\alpha$ and IL-6, while subcutaneous adipocytes secret one of the antiinflammatory cytokines such as adiponectin (37). A shift in the adipose tissue away from subcutaneous to visceral adipose sites with skeletal muscle loss during the aging process or cancer cachexia leads to an imbalance between proinflammatory adipokines and myokines $(15,38)$. This imbalance in the cytokine profile has been shown to lead to immunosenescence, particularity of the natural killer lymphocytes involved in the innate immunity $(14,39)$. On the other hand, visceral adiposity activates various kinds of immune cells through an increase in leptin and a decrease in adiponectin (14). Moreover, several studies have shown the effect of adiponectin and leptin on tumor cells; leptin promotes the growth and proliferation of tumor cells by activating various signaling pathways while adiponectin inhibits the oncogenic actions of leptin by blocking Stat 3 and Akt phosphorylation $(14,40)$. These results indicate that visceral adiposity with low muscularity would decrease the immune function for oncogenesis due to an imbalance in the adipokines and other cytokines, which could lead to an increased risk of death (14).

Therefore, introduction of prehabilitation strategy in sarcopenic and/or visceral obese HCC patients has to be considered. In fact, there is a few evidence that preoperative exercise and nutritional program may prevent nutritional deterioration, improved physical fitness before surgery, and shortened the postoperative hospital stay for the patients undergoing hepato-pancreas-biliary cancer or gastric cancer surgery $(41,42)$. However, further prospective studies are needed to investigate survival benefit of these programs.

The limitations of this study are as follows. First, this was a retrospective, single center study with a relatively 
small sample size especially in the sarcopenic group. Nevertheless, the sarcopenia and visceral obesity showed strong association with the overall survival of the HCC patients after surgical resection by multivariate Cox regression analysis. Second, this study did not measure frailty parameters such as muscle strength or performance. Nevertheless, this study is the first study demonstrating role of the 3 components of body composition defined by the PMI, PMA and VATI in the prognosis of curatively resected HCC patients in South Korea with reproducibly accurate measurements and a considerably long follow-up.

In conclusion, sarcopenia and visceral adiposity predicts a poor overall survival but not recurrence-free survival in HCC patients who underwent curative hepatic resection, while IMF deposition was not an independent factor for the overall survival. Therefore, enforced nutrition and exercise to correct sarcopenia and visceral adiposity may be helpful to HCC patients during the preoperative period. Further prospective studies are needed to investigate survival benefit through enforced nutrition and exercise in HCC patients.

\section{Acknowledgments}

We appreciate Hye In Mann, Da woon Jeong, and Taehyun Nam at Seoul National University of Bundang Hospital for their efforts to measure the psoas muscle area.

Funding: This study was supported by an intramural grant (02-2017-041) from the Seoul National University Bundang Hospital.

\section{Footnote}

Reporting Checklist: The authors have completed the REMARK reporting checklist. Available at http://dx.doi. org/10.21037/tcr-20-2974

Data Sharing Statement: Available at http://dx.doi. org/10.21037/tcr-20-2974

Conflicts of Interest: All authors have completed the ICMJE uniform disclosure form (available at http://dx.doi. org/10.21037/tcr-20-2974). The authors have no conflicts of interest to declare.

Ethical Statement: The authors are accountable for all aspects of the work in ensuring that questions related to the accuracy or integrity of any part of the work are appropriately investigated and resolved. The study was conducted in accordance with the Declaration of Helsinki (as revised in 2013). This study was approved by the Institutional Review Board of Seoul National University Bundang Hospital (B-1603-340-111) and individual consent for this retrospective analysis was waived.

Open Access Statement: This is an Open Access article distributed in accordance with the Creative Commons Attribution-NonCommercial-NoDerivs 4.0 International License (CC BY-NC-ND 4.0), which permits the noncommercial replication and distribution of the article with the strict proviso that no changes or edits are made and the original work is properly cited (including links to both the formal publication through the relevant DOI and the license). See: https://creativecommons.org/licenses/by-nc-nd/4.0/.

\section{References}

1. World Health Organization. Globocan 2018. In: International Agency for Research on Cancer, 2018.

2. Cancer Stat Facts: Liver and Intrahepatic Bile Duct Cancer. In: National Cancer Institute. Available online: https://seer.cancer.gov/statfacts/html/livibd.html

3. GBD 2013 Mortality and Causes of Death Collaborators. Global, regional, and national age-sex specific all-cause and cause-specific mortality for 240 causes of death, 19902013: a systematic analysis for the Global Burden of Disease Study 2013. Lancet 2015;385:117-71.

4. Carey EJ, Lai JC, Wang CW, et al. A multicenter study to define sarcopenia in patients with end-stage liver disease. Liver Transpl 2017;23:625-33.

5. Dodson RM, Firoozmand A, Hyder O, et al. Impact of sarcopenia on outcomes following intra-arterial therapy of hepatic malignancies. J Gastrointest Surg 2013;17:212332.

6. Ha Y, Kim D, Han S, et al. Sarcopenia Predicts Prognosis in Patients with Newly Diagnosed Hepatocellular Carcinoma, Independent of Tumor Stage and Liver Function. Cancer Res Treat 2018;50:843-51.

7. Harimoto N, Shirabe K, Yamashita YI, et al. Sarcopenia as a predictor of prognosis in patients following hepatectomy for hepatocellular carcinoma. Br J Surg 2013;100:1523-30.

8. Iritani S, Imai K, Takai K, et al. Skeletal muscle depletion is an independent prognostic factor for hepatocellular carcinoma. J Gastroenterol 2015;50:323-32.

9. Nishikawa H, Nishijima N, Enomoto H, et al. Prognostic significance of sarcopenia in patients with hepatocellular carcinoma undergoing sorafenib therapy. Oncol Lett 
2017;14:1637-47.

10. Voron T, Tselikas L, Pietrasz D, et al. Sarcopenia Impacts on Short- and Long-term Results of Hepatectomy for Hepatocellular Carcinoma. Ann Surg 2015;261:1173-83.

11. Chang KV, Chen JD, Wu WT, et al. Association between Loss of Skeletal Muscle Mass and Mortality and Tumor Recurrence in Hepatocellular Carcinoma: A Systematic Review and Meta-Analysis. Liver Cancer 2018;7:90-103.

12. Kamachi S, Mizuta T, Otsuka T, et al. Sarcopenia is a risk factor for the recurrence of hepatocellular carcinoma after curative treatment. Hepatol Res 2016;46:201-8.

13. Yabusaki N, Fujii T, Yamada S, et al. Adverse impact of low skeletal muscle index on the prognosis of hepatocellular carcinoma after hepatic resection. Int J Surg 2016; 30:136-42.

14. Fujiwara N, Nakagawa H, Kudo Y, et al. Sarcopenia, intramuscular fat deposition, and visceral adiposity independently predict the outcomes of hepatocellular carcinoma. J Hepatol 2015;63:131-40.

15. Hamaguchi Y, Kaido T, Okumura S, et al. Preoperative Visceral Adiposity and Muscularity Predict Poor Outcomes after Hepatectomy for Hepatocellular Carcinoma. Liver Cancer 2019;8:92-109.

16. Rabe C, Pilz T, Klostermann C, et al. Clinical characteristics and outcome of a cohort of 101 patients with hepatocellular carcinoma. World J Gastroenterol 2001;7:208-15.

17. Korean Liver Cancer Study Group (KLCSG); National Cancer Center, Korea (NCC). 2014 Korean Liver Cancer Study Group-National Cancer Center Korea practice guideline for the management of hepatocellular carcinoma. Korean J Radiol 2015;16:465-522.

18. Hamaguchi Y, Kaido T, Okumura S, et al. Proposal for new diagnostic criteria for low skeletal muscle mass based on computed tomography imaging in Asian adults. Nutrition 2016;32:1200-5.

19. Hiraoka A, Aibiki T, Okudaira T, et al. Muscle atrophy as pre-sarcopenia in Japanese patients with chronic liver disease: computed tomography is useful for evaluation. J Gastroenterol 2015;50:1206-13.

20. Mourtzakis M, Prado CM, Lieffers JR, et al. A practical and precise approach to quantification of body composition in cancer patients using computed tomography images acquired during routine care. Appl Physiol Nutr Metab 2008;33:997-1006.

21. Lausen B, Schumacher M. Maximally selected rank statistics. Biometrics 1992;48:73-85.
22. Prado CM, Lieffers JR, McCargar LJ, et al. Prevalence and clinical implications of sarcopenic obesity in patients with solid tumours of the respiratory and gastrointestinal tracts: a population-based study. Lancet Oncol 2008; 9:629-35.

23. Nishikawa H, Shiraki M, Hiramatsu A, et al. Japan Society of Hepatology guidelines for sarcopenia in liver disease (1st edition): Recommendation from the working group for creation of sarcopenia assessment criteria. Hepatol Res 2016;46:951-63.

24. Sakuma K, Aoi W, Yamaguchi A. Molecular mechanism of sarcopenia and cachexia: recent research advances. Pflugers Arch 2017;469:573-91.

25. Kitajima Y, Takahashi H, Akiyama T, et al. Supplementation with branched-chain amino acids ameliorates hypoalbuminemia, prevents sarcopenia, and reduces fat accumulation in the skeletal muscles of patients with liver cirrhosis. J Gastroenterol 2018;53:427-37.

26. Kim YS, Lee Y, Chung YS, et al. Prevalence of sarcopenia and sarcopenic obesity in the Korean population based on the Fourth Korean National Health and Nutritional Examination Surveys. J Gerontol A Biol Sci Med Sci 2012;67:1107-13.

27. Valero V 3rd, Amini N, Spolverato G, et al. Sarcopenia adversely impacts postoperative complications following resection or transplantation in patients with primary liver tumors. J Gastrointest Surg 2015;19:272-81.

28. Goodpaster BH, Carlson CL, Visser M, et al. Attenuation of skeletal muscle and strength in the elderly: The Health ABC Study. J Appl Physiol (1985) 2001;90:2157-65.

29. Hamaguchi Y, Kaido T, Okumura S, et al. Muscle Steatosis is an Independent Predictor of Postoperative Complications in Patients with Hepatocellular Carcinoma. World J Surg 2016;40:1959-68.

30. Aubrey J, Esfandiari N, Baracos VE, et al. Measurement of skeletal muscle radiation attenuation and basis of its biological variation. Acta Physiol (Oxf) 2014;210:489-97.

31. Kelley DE, Goodpaster BH, Storlien L. Muscle triglyceride and insulin resistance. Annu Rev Nutr 2002;22:325-46.

32. Gilsanz V, Kremer A, Mo AO, et al. Vitamin D status and its relation to muscle mass and muscle fat in young women. J Clin Endocrinol Metab 2010;95:1595-601.

33. Zoico E, Rossi A, Di Francesco V, et al. Adipose tissue infiltration in skeletal muscle of healthy elderly men: relationships with body composition, insulin resistance, and inflammation at the systemic and tissue level. J 
Gerontol A Biol Sci Med Sci 2010;65:295-9.

34. Fedirko V, Duarte-Salles T, Bamia C, et al. Prediagnostic circulating vitamin D levels and risk of hepatocellular carcinoma in European populations: a nested case-control study. Hepatology 2014;60:1222-30.

35. Nakagawa H, Fujiwara N, Tateishi R, et al. Impact of serum levels of interleukin-6 and adiponectin on all-cause, liver-related, and liver-unrelated mortality in chronic hepatitis C patients. J Gastroenterol Hepatol 2015; 30:379-88.

36. Nault JC, Pigneur F, Nelson AC, et al. Visceral fat area predicts survival in patients with advanced hepatocellular carcinoma treated with tyrosine kinase inhibitors. Dig Liver Dis 2015;47:869-76.

37. Marra F, Bertolani C. Adipokines in liver diseases. Hepatology 2009;50:957-69.

38. Tilg H, Moschen AR. Adipocytokines: mediators linking

Cite this article as: Jang HY, Choi GH, Hwang SH, Jang ES, Kim JW, Ahn JM, Choi Y, Cho JY, Han HS, Lee J, Chung JW, Baeg JY, Jeong SH. Sarcopenia and visceral adiposity predict poor overall survival in hepatocellular carcinoma patients after curative hepatic resection. Transl Cancer Res 2021;10(2):854-866. doi: 10.21037/tcr-20-2974 adipose tissue, inflammation and immunity. Nat Rev Immunol 2006;6:772-83.

39. Lutz CT, Quinn LS. Sarcopenia, obesity, and natural killer cell immune senescence in aging: altered cytokine levels as a common mechanism. Aging (Albany NY) 2012;4:535-46.

40. Sharma D, Wang J, Fu PP, et al. Adiponectin antagonizes the oncogenic actions of leptin in hepatocellular carcinogenesis. Hepatology 2010;52:1713-22.

41. Nakajima H, Yokoyama Y, Inoue T, et al. Clinical Benefit of Preoperative Exercise and Nutritional Therapy for Patients Undergoing Hepato-Pancreato-Biliary Surgeries for Malignancy. Ann Surg Oncol 2019;26:264-72.

42. Yamamoto K, Nagatsuma Y, Fukuda Y, et al. Effectiveness of a preoperative exercise and nutritional support program for elderly sarcopenic patients with gastric cancer. Gastric Cancer 2017;20:913-8. 